\title{
Density Functional Application to Strongly Correlated Electron Systems
}

\author{
H. Eschrig \\ K. Koepernik \\ I. Chaplygin
}

email: h.eschrig@ifw-dresden.de

Tel: $+49(0) 351-4659-380$

Fax: $+49(0) 351-4659-500$

This paper is dedicated to Walter Kohn on the occassion of his eightieth birthday in March 2003 


\begin{abstract}
The LSDA $+U$ approach to density functional theory is carefully reanalyzed. Its possible link to single-particle Green's function theory is occasionally discussed. A simple and elegant derivation of the important sum rules for the on-site interaction matrix elements linking them to the values of $U$ and $J$ is presented. All necessary expressions for an implementation of LSDA $+U$ into a non-orthogonal basis solver for the Kohn-Sham equations are given, and implementation into the FPLO solver [15] is made. Results of application to several planar cuprate structures are reported in detail and conclusions on the interpretation of the physics of the electronic structure of the cuprates are drawn.
\end{abstract}




\section{Introduction}

Density functional theory (DFT) based on the variational principle by Hohenberg and Kohn [1] has nowadays a rigorous mathematical basis, mainly due to work by Lieb [2. As a theory for (chosen) ground state properties of a many-particle system, it holds true for any Coulomb quantum system with arbitrarily strong correlations in the ground state. All hampering representability problems of the early time are gone (see for instance [3]). However, the central quantity, the universal density functional is not known, only its existence can be proved, and we have no fully systematic access by approximations. Hence, so far (and very likely also in future) we have to model it and to probe the models by comparison to phenomenology. This situation is not principally different from other manyparticle approaches where either models of sufficiently simple Hamiltonians are used (in quantum field theory) or the wave function is modeled (for instance in Hartree-Fock or Gutzwiller approaches).

The situation is even less satisfactory in solid state theory, if the focus is on the excitation spectra instead on the ground state, because in most cases the spectrum of the many-body Hamiltonian has no separate physical relevance at all except for its formal use in theoretical expressions for the partition function. Instead, what is measured are the spectra of various quasistationary excitations, defined from few-particle Green's functions, the self-energy parts of which, besides being energy dependent non-linear integral operators, are also density functionals.

Although this is not a principal restriction of DFT, the models in use so far (local (spin) density approximation, L(S)DA, in the following the acronym LSDA is used for both LDA and LSDA, generalized gradient approximation, GGA, LSDA plus self-interaction correction, SIC, LSDA plus onsite Coulomb repulsion, $\mathrm{LSDA}+U, \ldots$ ) are subject to the adiabatic approximation for the electron-lattice interaction.

DFT in the Kohn-Sham (KS) approach to solids yields a KS band structure, which as such does also not have a direct physical meaning. Instead, the quasiparticle band structure of Bloch electrons is obtained from the self-energy of the electron Green's function. It has become common use to speak of weak correlations, if in the vicinity of the Fermi level the LSDA KS potential and the electron self-energy are not very different. This does by no means imply that the correlation energy itself, defined as the difference between the true total energy and the Hartree-Fock energy, is small or much smaller than in strongly correlated systems (where the LSDA KS potential differs strongly from the electron selfenergy, the latter often jumps as a function of energy at the Fermi level). It is a general experience that in the latter cases also the ground state properties, or certain ground state properties, are much worse reproduced by the LSDA than in weakly correlated cases (in the above definition).

This paper deals with strongly correlated systems treated by means of the 
LSDA $+U$ approach. Maybe the first precursor of an LSDA $+U$ calculation (at that time not fully self-consistent) was the treatment of $4 f$-electrons by Herbst et al. 4]. A first fully self-consistent calculation of values of the Hubbard $U$ was performed by Dederichs et al. [5] for the $4 f$-states of Ce by applying the KoringaKohn-Rostoker solver of the KS equations to a constraint impurity problem. An early similar approach to $U$ for $\mathrm{NiO}$ by Norman and Freeman [6] used the APW solver in a super-cell formulation. For the further development of this subject see [7.

Pickett and Wang [8] and Hybertsen and Louie 9] based the so-called $G W$ approximation for the electron self-energy of semi-conductors and insulators on LDA results for the density and KS bands as a starting approximation for the $G W$-approach. In these cases the main difference between the LDA KS potential and the self-energy is a jump of the latter, constant in $\boldsymbol{r}$-space at the Fermi level (scissors operation). This is still considered a weakly correlated case, but these were the first estimates of the self-energy of an inhomogeneous situation as a density functional.

A systematic incorporation of the Hubbard $U$-potential into the DFT model functionals started with two papers by Anisimov at al. [10, 11. While it was proposed in [10] to model the total spin-dependence by the $U$-functional and to treat the spin independent functional by the LDA, in 11 the orbital polarization part ( $m$-dependent occupation of local orbitals) was treated by the $U$-functional and the isotropic (in $\boldsymbol{r}$-space) part of the spin-density was treated in LSDA. This has the advantage that spin polarization effects can be treated more generally, not only in the strongly correlated orbitals. However, this version, later on called 'around the mean field', AMF, by Czyżyk and Sawatzky [12, gives nearly nothing for a half-filled fully spin polarized shell as in $\mathrm{Mn}^{2+}$ or in Gd. Therefore, aiming mainly at reproducing the photoemission spectra (which essentially means modeling the electron self-energy rather that the KS potential), an alternative $U$-functional was introduced in [12] and called the 'atomic limit' version, AL. This version which roughly shifts unoccupied orbital energies upward by $U / 2$ and occupied orbital energies downward by $U / 2$ independent of the shell filling (even for filled and empty shells), has been widely used since. For a survey see [13.

It has to be confessed that all LSDA $+U$ models up to now depend on the basis used for the KS solver. Most results are obtained so far with an LMTO implementation. For a recent APW implementation see [14].

In this paper, in Section 2, an FPLO implementation is described. FPLO [15] is a high precision high efficiency $\mathrm{KS}$ solver which uses a minimum basis (and hence is fast) containing only local basis functions which are optimized in both a numerical and chemical sense. (It competes in accuracy with well converged full-potential APW.) After a short outline how $U$ is integrated into DFT, the correlated orbitals used in the FPLO implementation are introduced. Since literature statements [12] say that the most important sum rules for the 
interaction matrix elements (screened Slater integrals) of those orbitals are cumbersome to verify, a very simple and elegant derivation is given here. After the necessary analysis of the orbital occupation matrix for the non-orthogonal basis of FPLO and the introduction of the AMF and AL functionals in the FPLO implementation, explicit expressions for the $U$-potential and for the total energy are given as they are coded in FPLO LSDA $+U$. In Section 3, new applications to cuprate structures, the 'infinite layer' compound $\mathrm{CaCuO}_{2}$, the undoped singlelayer compound $\mathrm{Sr}_{2} \mathrm{CuO}_{2} \mathrm{Cl}_{2}$ and the bilayer high-temperature superconductor $\mathrm{Bi}_{2} \mathrm{Sr}_{2} \mathrm{CaCu}_{2} \mathrm{O}_{8}$ are considered and the results are compared to both magnetic ground state properties and photoemission spectra. A short summary is given in Section 4.

\section{The FPLO implementation of the LSDA $+U$ approach}

The underlying frame of the LSDA $+U$ approach is the Hohenberg-Kohn variational principle,

$$
E[\check{v}, N]=\min _{\check{n}}\left\{H[\check{n}]+\sum_{s s^{\prime}} \int d^{3} r v_{s s^{\prime}}(\boldsymbol{r}) n_{s^{\prime} s}(\boldsymbol{r}) \mid \sum_{s} \int d^{3} r n_{s s}(\boldsymbol{r})=N\right\}
$$

for the ground state energy $E$ and spin density $\check{n}=\left(n_{s s^{\prime}}\right)$ of $N$ electrons in an external spin dependent potential $\breve{v}$,

$$
\sum_{s s^{\prime}} \int d^{3} r v_{s s^{\prime}}(\boldsymbol{r}) n_{s^{\prime} s}(\boldsymbol{r})=\int d^{3} r(v n-\boldsymbol{B} \cdot \boldsymbol{m})
$$

which holds true in any case of arbitrarily strong correlation. It is based on many-particle quantum theory by rigorous mathematics. Of course, the density functional $H[\check{n}]$ is unknown.

The generalized Kohn-Sham modeling of this functional is by parameterizing the variational spin density by new variational parameters: the Kohn-Sham orbitals $\phi_{i}(\boldsymbol{r}, s)$ and orbital occupation numbers $n_{i}$,

$$
n_{s s^{\prime}}(\boldsymbol{r})=\sum_{i} \phi_{i}(\boldsymbol{r} s) n_{i} \phi_{i}^{*}\left(\boldsymbol{r} s^{\prime}\right), \quad\left\langle\phi_{i} \mid \phi_{j}\right\rangle=\delta_{i j}, \quad 0 \leq n_{i} \leq 1, \quad \sum_{i} n_{i}=N,
$$

and by splitting the density functional into an orbital variation expression $K[\check{n}]$ and a (possibly generalized by gradient terms) local density expression $L[\check{n}]$ :

$$
\begin{aligned}
& H[\check{n}]=K[\check{n}]+L[\check{n}] \\
& K[\check{n}]=\min _{\left\{\phi_{i}, n_{i}\right\}}\left\{k\left[\phi_{i}, n_{i}\right] \mid \sum_{i} \phi_{i} n_{i} \phi_{i}^{*}=\check{n}\right\} \\
& L[\check{n}]=\int d^{3} \operatorname{rn}(\boldsymbol{r}) l\left(n_{s s^{\prime}}(\boldsymbol{r}), \nabla n, \ldots\right) .
\end{aligned}
$$


This puts the Hohenberg-Kohn variational principle into the Kohn-Sham form

$$
\begin{aligned}
E[\check{v}, N]=\min _{\phi_{i}, n_{i}}\left\{k\left[\phi_{i}, n_{i}\right]+L\left[\sum_{i} \phi_{i} n_{i} \phi_{i}^{*}\right]+\sum_{i} n_{i}\left\langle\phi_{i}|\check{v}| \phi_{i}\right\rangle \mid\right. \\
\left.\qquad\left\langle\phi_{i} \mid \phi_{j}\right\rangle=\delta_{i j}, 0 \leq n_{i} \leq 1, \sum_{i} n_{i}=N\right\} .
\end{aligned}
$$

While for the $L$-functional LSDA or GGA models are in use, $k$ is modeled by $\mathrm{LSDA}$ or LSDA+SIC or LSDA $+U$. Variation of $\phi_{i}^{*}$ yields the generalized KohnSham equation,

$$
\left[\frac{1}{n_{i}} \frac{\delta k}{\delta \phi^{*}}+\check{v}^{L}+\check{v}\right] \phi_{i}=\phi_{i} \varepsilon_{i}, \quad v_{s s^{\prime}}^{L}=\frac{\delta L}{\delta n_{s^{\prime} s}},
$$

and variation of the $n_{i}$ yields the common aufbau principle which holds true for all model variants within this frame and which says that the ground state density is obtained by occupying the $N$ orbitals with the lowest $\varepsilon_{i}$.

The variants of the LSDA $+U$ model correspond to

$$
k=t+e^{\mathrm{H}}+e^{U}, \quad t+e^{\mathrm{H}}=\sum_{i} n_{i}\left\langle\phi_{i}|\hat{t}| \phi_{i}\right\rangle+\frac{1}{2} \sum_{i j} n_{i} n_{j}\left\langle\phi_{i} \phi_{j}\left|r_{i j}^{-1}\right| \phi_{i} \phi_{j}\right\rangle,
$$

where $e^{U}$ is expressed through projection onto correlated local orbitals $\left.\mid \boldsymbol{R} \mu \sigma\right)$ centered at site (or in the unit cell) $\boldsymbol{R}$ and with orbital and spin quantum numbers $\mu$ and $\sigma$. The projection is given by a local orbital occupation number $\tilde{n}_{\mu \sigma}$ which depends on the variational quantities $\phi_{i}, n_{i}$ :

$$
e^{U}=e^{U}\left(\tilde{n}_{\mu \sigma}\left[\phi_{i}, n_{i}\right]\right), \quad \frac{1}{n_{i}} \frac{\delta}{\delta \phi_{i}^{*}} e_{U}=\sum_{\boldsymbol{R} \mu \sigma} \frac{\partial e^{U}}{\partial \tilde{n}_{\mu \sigma}} \frac{1}{n_{i}} \frac{\delta \tilde{n}_{\mu \sigma}}{\delta \phi_{i}^{*}} .
$$

The functional derivative on the r.h.s. of the last expression yields the projection while the partial derivative defines the orbital and spin dependent $U$-potential: $v_{\mu \sigma}^{U}=\partial e^{U} / \partial \tilde{n}_{\mu \sigma}$. It is crucial for fitting the models in use into the general Hohenberg-Kohn-Sham frame that the correlated orbitals themselves as well as the actual value of $U$ are understood fixed and not variational although they may be context dependent. They kind of define a location, relevant in a given context, in the variational functional space and a functional contribution from that location.

\subsection{Correlated orbitals}

In the so called rotationally invariant LSDA $+U$ approach the correlated local orbitals are assumed to be angular momentum eigenstates centered at $\boldsymbol{R}$, say, 
with predefined orbital and spin momentum quantization axes (which both need not be the same)

$$
\left.\mid \boldsymbol{R}_{i} m_{i} \sigma_{i}\right), \quad m_{i}=-l_{i}, \ldots, l_{i}, \quad \sigma_{i}=\uparrow, \downarrow .
$$

Only one-site matrix elements, $\boldsymbol{R}_{1}=\boldsymbol{R}_{2}=\boldsymbol{R}_{3}=\boldsymbol{R}_{4}$, are considered:

$$
\left(m_{1} m_{2}|\tilde{w}| m_{3} m_{4}\right), \quad \tilde{w} \approx \tilde{w}\left(\left|\boldsymbol{r}-\boldsymbol{r}^{\prime}\right|\right), \quad \sigma_{1}=\sigma_{3}, \sigma_{2}=\sigma_{4}
$$

The rotational invariance refers to the screened electron-electron interaction, $\tilde{w}$, which is of course an approximation as regards the screening. As a consequence, the $\mathrm{SO}_{3}$ transformation properties of the matrix elements are

$$
\begin{aligned}
&\left(m_{1} m_{2}|\tilde{w}| m_{3} m_{4}\right)=\sum_{m_{1}^{\prime} m_{2}^{\prime} m_{3}^{\prime} m_{4}^{\prime}} U_{m_{1} m_{1}^{\prime}}^{\dagger}(\hat{O}) U_{m_{2} m_{2}^{\prime}}^{\dagger}(\hat{O}) * \\
& *\left(m_{1}^{\prime} m_{2}^{\prime}|\tilde{w}| m_{3}^{\prime} m_{4}^{\prime}\right) U_{m_{3}^{\prime} m_{3}}(\hat{O}) U_{m_{4}^{\prime} m_{4}}(\hat{O}),
\end{aligned}
$$

where $\hat{O}$ is any rotation of the $\boldsymbol{r}$-space and the $U$-matrices (not to be confused with the Coulomb integral $U$ ) yield the relevant $\mathrm{SO}_{3}$ representation:

$$
\begin{gathered}
U^{\dagger}(\hat{O}) U(\hat{O})=1=U(\hat{O}) U^{\dagger}(\hat{O}) \\
\int d \hat{O} U_{m_{1} m_{2}}(\hat{O}) U_{m_{3} m_{4}}^{\dagger}(\hat{O})=\frac{1}{2 l+1} \delta_{m_{1} m_{4}} \delta_{m_{2} m_{3}}
\end{gathered}
$$

In the last orthogonality relation, $d \hat{O}$ is Haar's measure of the $S_{3}, \int d \hat{O}=1$.

These fundamental representation properties allow for a very simple and elegant derivation of the important sum rules for the matrix elements: Use unitarity of $U$ and integrate over $d \hat{O}$ to obtain

$$
\begin{aligned}
& \sum_{m_{1}}\left(m_{1} m_{2}|\tilde{w}| m_{1} m_{4}\right)= \\
& =\sum_{m_{1}} \sum_{m_{1}^{\prime} m_{2}^{\prime} m_{3}^{\prime} m_{4}^{\prime}} U_{m_{1} m_{1}^{\prime}}^{\dagger}(\hat{O}) U_{m_{2} m_{2}^{\prime}}^{\dagger}(\hat{O})\left(m_{1}^{\prime} m_{2}^{\prime}|\tilde{w}| m_{3}^{\prime} m_{4}^{\prime}\right) \cdot \\
& =\sum_{m_{1}^{\prime} m_{2}^{\prime} m_{3}^{\prime} m_{4}^{\prime}} \delta_{m_{1}^{\prime} m_{3}^{\prime}} U_{m_{2} m_{2}^{\prime} m_{1}}^{\dagger}(\hat{O}) U_{m_{4}^{\prime} m_{4}}(\hat{O})= \\
& =\frac{1}{2 l+1} \sum_{m_{1}^{\prime} m_{2}^{\prime} m_{4}^{\prime}}\left(m_{1}^{\prime} m_{2}^{\prime}|\tilde{w}| m_{3}^{\prime} m_{4}^{\prime}\right) U_{m_{4}^{\prime} m_{4}}(\hat{O})= \\
& =\frac{\delta_{m_{2} m_{4}}}{2 l+1} \sum_{m_{1}^{\prime} m_{2}^{\prime}}\left(m_{1}^{\prime} m_{2}^{\prime}|\tilde{w}| m_{1}^{\prime} m_{4}^{\prime}\right) \delta_{m_{2} m_{4}} \delta_{m_{2}^{\prime} m_{4}^{\prime}}=
\end{aligned}
$$

The last equation is the definition of the Coulomb integral $U$. In the same 
manner,

$$
\begin{aligned}
& \sum_{m_{1}}\left(m_{1} m_{2}|\tilde{w}| m_{3} m_{1}\right)= \\
& =\frac{\delta_{m_{2} m_{3}}}{2 l+1} \sum_{m_{1}^{\prime} m_{2}^{\prime}}\left(m_{1}^{\prime} m_{2}^{\prime}|\tilde{w}| m_{2}^{\prime} m_{1}^{\prime}\right)=\delta_{m_{2} m_{3}}(U+2 l J)
\end{aligned}
$$

is obtained which additionally defines the exchange integral $J$. The first result (13) is intuitively obvious: after summation over $m_{1}$ and integration over $\boldsymbol{r}$ in the matrix element, no angular dependence with respect to $\boldsymbol{r}^{\prime}$ is left except the orthogonality $\left(m_{2} \mid m_{4}\right)=\delta_{m_{2} m_{4}}$. The second result (14) is less obvious but nevertheless true.

Expansion of the interaction function into spherical harmonics,

$$
\begin{aligned}
& \tilde{w}\left(\left|\boldsymbol{r}_{1}-\boldsymbol{r}_{2}\right|\right)=\tilde{w}\left(\left(r_{1}^{2}+r_{2}^{2}-2 r_{1} r_{2} \cos \theta\right)^{1 / 2}\right)= \\
& =\sum_{l=0}^{\infty} \tilde{w}_{l}\left(r_{1}, r_{2}\right) P_{l}(\cos \theta)=\sum_{l=0}^{\infty} \tilde{w}_{l}\left(r_{1}, r_{2}\right) \frac{4 \pi}{2 l+1} \sum_{m=-l}^{l} Y_{l m}\left(\hat{\boldsymbol{r}}_{1}\right) Y_{l m}^{*}\left(\hat{\boldsymbol{r}}_{2}\right)
\end{aligned}
$$

leads to Slater's analysis

$$
\begin{aligned}
\left(m_{1} m_{2}|\tilde{w}| m_{3} m_{4}\right) & =\sum_{l=0}^{2 l_{i}} \tilde{F}_{l} a_{l}\left(m_{1} m_{2} m_{3} m_{4}\right), \\
\tilde{F}_{l} & =\iint_{0}^{\infty} d r_{1} d r_{2}\left(r_{1} R_{i}\left(r_{1}\right)\right)^{2}\left(r_{2} R_{i}\left(r_{2}\right)\right)^{2} \tilde{w}_{l}\left(r_{1}, r_{2}\right) \\
& \approx \iint_{0}^{\infty} d r_{1} d r_{2}\left(r_{1} R_{i}\left(r_{1}\right)\right)^{2}\left(r_{2} R_{i}\left(r_{2}\right)\right)^{2} \frac{r_{<}^{l}}{r_{>}^{l+1}} \text { for } l>0, \\
a_{l}\left(m_{1} m_{2} m_{3} m_{4}\right) & =\frac{4 \pi}{2 l+1} \sum_{m=-l}^{l}\left(Y_{l_{i} m_{1}}\left|Y_{l m}\right| Y_{l_{i} m_{3}}\right)\left(Y_{l_{i} m_{4}}\left|Y_{l m}\right| Y_{l_{i} m_{2}}\right)^{*} .
\end{aligned}
$$

Here, $l_{i}$ is the angular momentum of the considered shell, and the second line for $\tilde{F}_{l}$ holds for the unscreened Coulomb interaction which for $l>0$ is a reasonable approximation since intraatomic screening is effective only for the $s$-component of the interaction.

Now, from $\sum_{m} Y_{l m}(\boldsymbol{r}) Y_{l m}^{*}(\boldsymbol{r})=P_{l}(1)(2 l+1) / 4 \pi$ and

$$
\begin{aligned}
& \sum_{m_{1}} a_{l}\left(m_{1} m_{2} m_{1} m_{2}\right)= \\
& =\frac{4 \pi}{2 l+1}\left[\sum_{m_{1}}\left(Y_{l_{i} m_{1}}\left|Y_{l 0}\right| Y_{l_{i} m_{1}}\right)\right]\left(Y_{l_{i} m_{2}}\left|Y_{l 0}\right| Y_{l_{i} m_{2}}\right)^{*}= \\
& =\sqrt{4 \pi} \frac{2 l_{i}+1}{2 l+1} \delta_{l 0}\left(Y_{l_{i} m_{2}}\left|Y_{l 0}\right| Y_{l_{i} m_{2}}\right)^{*}=\left(2 l_{i}+1\right) \delta_{l 0}
\end{aligned}
$$


it follows immediately that

$$
U=\tilde{F}_{0}
$$

Furthermore,

$$
\begin{aligned}
\sum_{m_{1} m_{2}} a_{l}\left(m_{1} m_{2} m_{2} m_{1}\right) & =\frac{4 \pi}{2 l+1} \sum_{m_{1} m_{2}}\left(Y_{l_{i} m_{1}}\left|Y_{l m}\right| Y_{l_{i} m_{2}}\right)\left(Y_{l_{i} m_{2}}\left|Y_{l m}^{*}\right| Y_{l_{i} m_{1}}\right)= \\
& =\frac{4 \pi}{2 l+1} \iint d \Omega_{1} d \Omega_{2}\left(\sum_{m_{1}} Y_{l_{i} m_{1}}\left(\boldsymbol{r}_{2}\right) Y_{l_{i} m_{1}}^{*}\left(\boldsymbol{r}_{1}\right)\right) * \\
& *\left(\sum_{m_{2}} Y_{l_{i} m_{2}}\left(\boldsymbol{r}_{1}\right) Y_{l_{i} m_{2}}^{*}\left(\boldsymbol{r}_{2}\right)\right)\left(\sum_{m} Y_{l m}\left(\boldsymbol{r}_{1}\right) Y_{l m}^{*}\left(\boldsymbol{r}_{2}\right)\right)= \\
& =\frac{\left(2 l_{i}+1\right)^{2}}{(4 \pi)^{2}} \iint d \Omega_{1} d \Omega_{2}\left[P_{l_{i}}\left(\cos \theta_{12}\right)\right]^{2} P_{l}\left(\cos \theta_{12}\right)= \\
& =\frac{\left(2 l_{i}+1\right)^{2}}{4 \pi} \int d \Omega\left[P_{l_{i}}(\cos \theta)\right]^{2} P_{l}(\cos \theta)= \\
& =\left(2 l_{i}+1\right)^{2}\left(\begin{array}{ccc}
l_{i} & l & l_{i} \\
0 & 0 & 0
\end{array}\right)^{2}
\end{aligned}
$$

and hence

$$
\begin{aligned}
\sum_{m_{1} m_{2}}\left(m_{1} m_{2}|\tilde{w}| m_{2} m_{1}\right) & =\left(2 l_{i}+1\right)^{2} \sum_{l=0}^{2 l_{i}} \tilde{F}_{l}\left(\begin{array}{ccc}
l_{i} & l & l_{i} \\
0 & 0 & 0
\end{array}\right)^{2}= \\
& =\left(2 l_{i}+1\right)\left(U+2 l_{i} J\right) .
\end{aligned}
$$

Eqs. (17) and (18) relate the Coulomb and exchange integrals $U$ and $J$ to Slater's (screened) integrals $\tilde{F}_{l}$.

Recall, however, that the whole analysis presupposes the isotropy of screening which could be questioned at least in cases of strong directional covalency.

\subsection{The orbital occupation matrix}

The variants of the LSDA $+U$ model are all depending on the basis set of the solver of the Kohn-Sham equations. There are a few subtleties in this game which never have been discussed in the literature. Here, a non-orthogonal local basis implementation [15] of the solver will be used, since a local orbital representation is mandatory for considering strong correlations. Non-orthogonality of the basis is rather the rule than the exception for high precision solvers. For an LMTO solver see [16, for an LAPW solver see [14.

Consider Kohn-Sham orbitals $|k\rangle=\left|\phi_{k}\right\rangle$ and orbital occupation numbers $n_{k}$ as previously; they need not be eigenstates of spin. Let $\left.\{\mid l)\right\}$ be a possibly non-orthogonal basis for Kohn-Sham orbitals: $\left.|k\rangle=\sum_{l} \mid l\right) c_{l k}, S_{l l^{\prime}}=\left(l \mid l^{\prime}\right)$. (Systematically, brackets are used for the Kohn-Sham orbitals and parentheses for 
the local basis orbitals.) For an orthogonal projection onto those basis orbitals the contragredient basis $\left.\mid l\}=\sum_{l^{\prime}} \mid l^{\prime}\right)\left(S^{-1}\right)_{l^{\prime} l}, \quad\left\{l \mid l^{\prime}\right)=\delta_{l l^{\prime}}$ is needed. With its help, the occupation matrix $\tilde{n}=\tilde{n}\left[\phi_{k}, n_{k}\right]$ of correlated orbitals $\left.\mid m \sigma\right)$ at site $\boldsymbol{R}$ in an orthogonal form is introduced as

$$
\begin{aligned}
\tilde{n}_{m m^{\prime} \sigma} & =\sum_{k} \sum_{l l^{\prime}}\left(S^{-1}\right)_{(\boldsymbol{R} m \sigma), l}\left(l|k\rangle n_{k}\langle k| l^{\prime}\right)\left(S^{-1}\right)_{l^{\prime},\left(\boldsymbol{R} m^{\prime} \sigma\right)}= \\
& =\sum_{k} c_{(\boldsymbol{R} m \sigma), k} n_{k} c_{\left(\boldsymbol{R} m^{\prime} \sigma\right), k}^{*} .
\end{aligned}
$$

As usually it is assumed that the spin dependence can be made site diagonal by choosing a suitable spin quantization axis. The orbital occupation matrix may be diagonalized with respect to $m, m^{\prime}$ at each lattice site $\boldsymbol{R}$ and for each spin value $\sigma$ independently:

$$
\tilde{n}_{m m^{\prime} \sigma}=\tilde{U}_{m \mu_{\sigma}}^{(\sigma)} \tilde{n}_{\mu \sigma} \tilde{U}_{m^{\prime} \mu_{\sigma}}^{(\sigma) *}
$$

Averages over a correlated shell of angular momentum $l$,

$$
\tilde{n}_{\sigma}=\frac{1}{2 l+1} \sum_{\mu} \tilde{n}_{\mu \sigma}, \quad \tilde{n}=\frac{1}{2}\left(\tilde{n}_{\uparrow}+\tilde{n}_{\downarrow}\right),
$$

are used later on.

The projector in (8) is now

$$
\left.\frac{1}{n_{k}} \frac{\delta \tilde{n}_{\mu \sigma}}{\delta\langle k|}=\sum_{l l^{\prime}} \mid l^{\prime}\right)\left(S^{-1}\right)_{l^{\prime},(\boldsymbol{R} \mu \sigma)}\left(S^{-1}\right)_{(\boldsymbol{R} \mu \sigma), l}(l|k\rangle
$$

Naturally, in applications the correlated orbitals are assumed to form a subset of the basis orbitals, although this is not mandatory. In the FPLO scheme, the basis is adjusted in the course of iterations for solving the non-linear Kohn-Sham equations. This does not mean that the basis itself is treated as variational. Rather the relevant sector of the variational space is tracked along the way of search for the Kohn-Sham minimum. Likewise, the relevant location of correlation, that is the correlated orbitals as part of the basis, is tracked along.

\subsection{The Orbital Polarization LSDA $+U$ Functional}

This functional was introduced under the name 'around the mean field' (AMF) in Ref. [12. It is zero if the orbitals of an atomic shell are equally occupied, hence it depends on orbital polarization. It is given by

$$
l\left(n_{s s^{\prime}}(\boldsymbol{r}), \ldots\right)=l_{\mathrm{LSDA}},
$$




$$
\begin{aligned}
& e^{U, \mathrm{AMF}}=\frac{1}{2} \sum_{\boldsymbol{R} \sigma \mu \mu^{\prime}}\left\{\left(\mu_{\sigma} \mu_{-\sigma}^{\prime}|\tilde{w}| \mu_{\sigma} \mu_{-\sigma}^{\prime}\right)\left(\tilde{n}_{\mu \sigma}-\tilde{n}_{\sigma}\right)\left(\tilde{n}_{\mu^{\prime}-\sigma}-\tilde{n}_{-\sigma}\right)+\right. \\
& \left.+\left[\left(\mu_{\sigma} \mu_{\sigma}^{\prime}|\tilde{w}| \mu_{\sigma} \mu_{\sigma}^{\prime}\right)-\left(\mu_{\sigma} \mu_{\sigma}^{\prime}|\tilde{w}| \mu_{\sigma}^{\prime} \mu_{\sigma}\right)\right]\left(\tilde{n}_{\mu \sigma}-\tilde{n}_{\sigma}\right)\left(\tilde{n}_{\mu^{\prime} \sigma}-\tilde{n}_{\sigma}\right)\right\} \\
& =\frac{1}{2} \sum_{\boldsymbol{R} \sigma \mu \mu^{\prime}}\left\{\begin{array}{c}
\left(\mu_{\sigma} \mu_{-\sigma}^{\prime}|\tilde{w}| \mu_{\sigma} \mu_{-\sigma}^{\prime}\right) \tilde{n}_{\mu \sigma} \tilde{n}_{\mu^{\prime}-\sigma}+ \\
+\left[\left(\mu_{\sigma} \mu_{\sigma}^{\prime}|\tilde{w}| \mu_{\sigma} \mu_{\sigma}^{\prime}\right)-\left(\mu_{\sigma} \mu^{\prime} \mid\right.\right.
\end{array}\right. \\
& \left.+\left[\left(\mu_{\sigma} \mu_{\sigma}^{\prime}|\tilde{w}| \mu_{\sigma} \mu_{\sigma}^{\prime}\right)-\left(\mu_{\sigma} \mu_{\sigma}^{\prime}|\tilde{w}| \mu_{\sigma}^{\prime} \mu_{\sigma}\right)\right] \tilde{n}_{\mu \sigma} \tilde{n}_{\mu \sigma}\right\} \\
& -\frac{1}{2} \sum_{\boldsymbol{R} \sigma}\left\{U\left(N-\tilde{n}_{\sigma}\right)-J\left(N_{\sigma}-\tilde{n}_{\sigma}\right)\right\} N_{\sigma}, \\
& N_{\sigma}=\sum_{\mu} \tilde{n}_{\mu \sigma}=(2 l+1) \tilde{n}_{\sigma} .
\end{aligned}
$$

Here, $N$ is the number of electrons occupying a whole correlated $l$-shell, $N_{\sigma}$ is that for one spin sort. There is no danger of confusing it with the total electron number in Eqs. (105), the latter does not appear any more in the sequel. In the second equality use of the sum rules for the matrix elements was made.

The corresponding $U$-potential is, again most easily with use of the sum rules,

$$
\begin{aligned}
\frac{\partial e^{U, \mathrm{AMF}}}{\partial \tilde{n}_{\mu \sigma}}=\sum_{\mu^{\prime}}\left\{\left(\mu_{\sigma} \mu_{-\sigma}^{\prime}|\tilde{w}| \mu_{\sigma} \mu_{-\sigma}^{\prime}\right)\left(\tilde{n}_{\mu^{\prime}-\sigma}-\tilde{n}_{-\sigma}\right)+\right. & \\
& \left.+\left[\left(\mu_{\sigma} \mu_{\sigma}^{\prime}|\tilde{w}| \mu_{\sigma} \mu_{\sigma}^{\prime}\right)-\left(\mu_{\sigma} \mu_{\sigma}^{\prime}|\tilde{w}| \mu_{\sigma}^{\prime} \mu_{\sigma}\right)\right]\left(\tilde{n}_{\mu^{\prime} \sigma}-\tilde{n}_{\sigma}\right)\right\}
\end{aligned}
$$

One weak point of this version is that it yields no contribution at all in case of orbital independent occupation numbers $\tilde{n}_{\mu^{\prime} \sigma}=\tilde{n}_{\sigma}$. This is for instance the case of a half-filled completely spin polarized shell (e.g. $4 f$-shell of Gd). In the Gd case this is at least not too bad, as the LSDA gives nearly the right spin polarization energy of $\mathrm{Gd}$, although there is a problem with the right magnetic ground state (obtained antiferromagnetic in LSDA).

\subsection{The 'Atomic Limit' LSDA $+U$ Functional}

With the Gd case in mind and aiming at a better description of the photoelectron spectra, Czyżyk and Sawatzky introduced another functional in [12] which they labeled 'atomic limit' (AL). At least regarding its relation to photoemission it should rather be considered a model for the quasiparticle self-energy $\Sigma$ instead of being related to $e^{U}$. Nevertheless it was given in an $e^{U}$-form as (again 


$$
\begin{aligned}
& \left.l\left(n_{s s^{\prime}}(\boldsymbol{r}), \ldots\right)=l_{\mathrm{LSDA}}\right) \\
& e^{U, \mathrm{AL}}=\frac{1}{2} \sum_{\boldsymbol{R} \sigma \mu \mu^{\prime}}\left\{\begin{array}{c}
\left(\mu_{\sigma} \mu_{-\sigma}^{\prime}|\tilde{w}| \mu_{\sigma} \mu_{-\sigma}^{\prime}\right) \tilde{n}_{\mu \sigma} \tilde{n}_{\mu^{\prime}-\sigma}+ \\
\left.+\left[\left(\mu_{\sigma} \mu_{\sigma}^{\prime}|\tilde{w}| \mu_{\sigma} \mu_{\sigma}^{\prime}\right)-\left(\mu_{\sigma} \mu_{\sigma}^{\prime}|\tilde{w}| \mu_{\sigma}^{\prime} \mu_{\sigma}\right)\right] \tilde{n}_{\mu \sigma} \tilde{n}_{\mu \sigma}\right\}
\end{array}\right. \\
& \quad-\frac{1}{2} \sum_{\boldsymbol{R}}\left\{U N(N-1)-J \sum_{\sigma} N_{\sigma}\left(N_{\sigma}-1\right)\right\},= \\
& =e^{U, \mathrm{AMF}}+\frac{1}{2} \sum_{\boldsymbol{R} \sigma}(U-J)\left(1-\tilde{n}_{\sigma}\right) N_{\sigma} .
\end{aligned}
$$

The corresponding $U$-potential is

$$
\frac{\partial e^{U, \mathrm{AL}}}{\partial \tilde{n}_{\mu \sigma}}=\frac{\partial e^{U, \mathrm{AMF}}}{\partial \tilde{n}_{\mu \sigma}}-(U-J)\left(\tilde{n}_{\sigma}-\frac{1}{2}\right) .
$$

One characteristic feature of this $U$-potential is that in case of an isolated shell it moves the occupied states downward by $(U-J) / 2$ and the unoccupied states upward by $(U-J) / 2$ independent of the shell occupation. By way of contrast, the center of the AMF spin subshell potential split moves up with increasing subshell occupation (so that the shift of the occupied levels is zero in the case of a filled spin subshell and likewise the shift of the unoccupied levels of an empty spin subshell; this way yielding no shift at all in Gd.) On the other hand, the AL $4 f$-level splitting of Gd is approximately doubled compared to LSDA which is a rather good result in the sense of a self-energy correction.

\subsection{The Kohn-Sham Hamiltonian matrix element and the total energy}

Here the formulas are presented, which actually are implemented. For the sake of simplicity, the correlated orbitals are identified with selected local basis orbitals as discussed at the beginning of subsection 2.2. The diagonalization of the occupation matrix, although greatly simplifying the analytical derivations, does not have advantages when coded, since anyway the diagonalizing transformation is site- and spin-dependent. Therefore, the full occupation matrix $\tilde{n}_{m m^{\prime} \sigma}$ is kept. (In the following, the site index of all quantities is dropped. If multiple sites with correlated states are needed, the formulas apply to all sites separately.) The matrix is obtained from the KS states.

$$
\tilde{n}_{m m^{\prime} \sigma}=\sum_{n \boldsymbol{k}} c_{m \sigma}^{n \boldsymbol{k}} n_{n \boldsymbol{k} \sigma} c_{m^{\prime} \sigma}^{n \boldsymbol{k} *}
$$

Integration is over the irreducible part of the Brillouin zone and a symmetrization projector is applied afterwards to get the result for the full zone. 
The AMF $U$-potential matrix as given in the text after Eq. (8) becomes

$$
v_{m^{\prime} m \sigma}^{\mathrm{AMF}}=\sum_{\sigma^{\prime}} \sum_{\mu \mu^{\prime}}\left(\tilde{n}_{\mu \mu^{\prime} \sigma^{\prime}}-\tilde{n}_{\sigma^{\prime}} \delta_{\mu \mu^{\prime}}\right)\left[\left(m^{\prime} \mu^{\prime}|\tilde{w}| m \mu\right)-\delta_{\sigma \sigma^{\prime}}\left(m^{\prime} \mu^{\prime}|\tilde{w}| \mu m\right)\right]
$$

with the property $\operatorname{Tr} v_{m m^{\prime} \sigma}^{\mathrm{AMF}} \equiv 0$ for each $\sigma$ separately. There is no such property of the AL potential since here the up and down shifts are independent of the shell occupation. The interaction matrix elements are taken to be spin independent. They are calculated from the Slater parameters according to Eq. (16). (Recall that the $\tilde{F}_{i}$ are external parameters, not variational.)

The projection part of Eq. (8) gives only Kronecker deltas. Thus the matrix elements of the KS equation are modified by $v_{m m^{\prime} \sigma}^{\mathrm{AMF}}$ for every block of correlated orbitals. From the eigenvalues the band structure energy $E^{B}$ is calculated as usual. The kinetic energy is obtained from it by subtracting double counting corrections. Besides the LSDA corrections the band energy contains a term

$$
\Delta T^{\mathrm{AMF}}=\sum_{m m^{\prime} \sigma} v_{m^{\prime} m \sigma}^{\mathrm{AMF}} \tilde{n}_{m m^{\prime} \sigma} .
$$

Since the $U$-potential matrix is traceless, a constant diagonal term may be added to the occupation matrix to obtain

$$
\Delta T^{\mathrm{AMF}}=\sum_{m m^{\prime} \sigma} v_{m^{\prime} m \sigma}^{\mathrm{AMF}}\left(\tilde{n}_{m m^{\prime} \sigma}-\tilde{n}_{\sigma} \delta_{m m^{\prime}}\right)=2 E^{U, \mathrm{AMF}} .
$$

Finally, the LSDA $+U$, kinetic and total energy are

$$
\begin{aligned}
E^{U, \mathrm{AMF}} & =\frac{1}{2} \Delta T^{\mathrm{AMF}} \\
T & =T^{\mathrm{LSDA}}-2 E^{U, \mathrm{AMF}} \\
E & =E^{\mathrm{LSDA}}-E^{U, \mathrm{AMF}} .
\end{aligned}
$$

The $U^{\mathrm{AL}}$-potential matrix becomes

$$
v_{m^{\prime} m \sigma}^{\mathrm{AL}}=\sum_{\sigma^{\prime}} \sum_{\mu \mu^{\prime}} \tilde{n}_{\mu \mu^{\prime} \sigma^{\prime}}\left[\left(m^{\prime} \mu^{\prime}|\tilde{w}| m \mu\right)-\delta_{\sigma \sigma^{\prime}}\left(m^{\prime} \mu^{\prime}|\tilde{w}| \mu m\right)\right]-v_{\sigma}^{\mathrm{dc}} \delta_{m m^{\prime}}
$$

with

$$
v_{\sigma}^{\mathrm{d} c}=U\left(N-\frac{1}{2}\right)-J\left(N_{\sigma}-\frac{1}{2}\right)
$$

Again, every block belonging to a correlated orbital in the Hamilton matrix is modified by adding $v_{m m^{\prime} \sigma}^{\mathrm{AL}}$.

Using a nutritive zero it can be shown that

$$
\Delta T^{\mathrm{AL}} \equiv \sum_{m m^{\prime} \sigma} v_{m^{\prime} m \sigma}^{\mathrm{AL}} \tilde{n}_{m m^{\prime} \sigma}=2 E^{U, \mathrm{AL}}-\frac{U-J}{2} N
$$


Thus, the final result is

$$
\begin{aligned}
E^{U, \mathrm{AL}} & =\frac{1}{2} \Delta T^{\mathrm{AL}}+\frac{1}{2} \frac{U-J}{2} N \\
T & =T^{\mathrm{LSDA}}-2 E^{U, \mathrm{AL}}+\frac{U-J}{2} N \\
E & =E^{\mathrm{LSDA}}-E^{U, \mathrm{AL}}+\frac{U-J}{2} N .
\end{aligned}
$$

\section{Applications to Cuprates}

The structure of the 2D cuprates considered here may be described by the formula $\mathrm{B}\left(\mathrm{CuO}_{2}\right)_{n} \mathrm{Ca}_{n-1}$, where $\mathrm{B}$ denotes the block layer which separates stacks of $n$ $\mathrm{CuO}_{2}$-planes with $\mathrm{Ca}$ planes sandwiched in between.

The LSDA $+U$ approach was applied to three compounds $\left(\mathrm{CaCuO}_{2}(n=\infty)\right.$, $\mathrm{Sr}_{2} \mathrm{CuO}_{2} \mathrm{Cl}_{2}(n=1)$ and $\left.\mathrm{Bi}_{2} \mathrm{Sr}_{2} \mathrm{CaCu}_{2} \mathrm{O}_{8}(n=2)\right)$ with the focus on the orbital analysis of relevant bands. While the bandstructure gap between occupied and unoccupied bands is a ground state property (jump of the chemical potential as function of the particle number at zero temperature), the whole bandstructure refers to the excitation spectrum, and the KS bandstructure need not compare to photoemission, say. Nevertheless, although there is no deeper reason that the best LSDA $+U$ potential for the KS equations should be close to the electron selfenergy, there is some hope that like for weakly correlated systems the LSDA $+U$ bandstructure could again provide also an approximation to the quasi-particle spectrum. One should, however, be aware that in principle the best KS $U$-value need not be the same as the best $U$-value in Hubbard-type model Hamiltonians. The latter value should for instance be used in dynamical mean-field theory which is an approach to the electron Green's function (self-energy). A satisfactory link of the $U$-value to the $\mathrm{KS}$ variational quantities, the KS orbitals and KS orbital occupation numbers, is still missing. For LSDA $+U$ as a Hohenberg-Kohn model the most relevant results are ground state properties as structural parameters (lattice constants, Wyckoff parameters), magnetic structure and magnetic polarization energy and band gap. The lattice parameters for the cuprates are obtained in the usual \pm 2 p.c. agreement with experiment. They will not be considered in the following.

The FPLO version used is 3.00-5 [17. Here, the program settings are summarized, which are unique to all calculations. The LSDA version is that of Perdew and Zunger [18. The $\mathrm{Cu} 3 d$ orbitals are taken to be the correlated orbitals. For the sake of comparison, the same Slater parameters were used for all calculations: $U=8.16 \mathrm{eV}(0.3$ Hartree $), J=1 \mathrm{eV}\left(F_{2}=9 \mathrm{eV}, F_{4}=5 \mathrm{eV}\right)$. The orbitals were optimized in the non-magnetic structures and the resulting compression radii were used also for the antiferromagnetic (AFM) LSDA- $U$ calculations. Unless explicitly else stated the "around mean field" (AMF) functional is used. 


\section{$3.1 \quad \mathrm{CaCuO}_{2}$}

The 'infinite layer' cuprate $\mathrm{CaCuO}_{2}$ (no block layer B) does not exist in nature, but there is an isostructural compound $\mathrm{Ca}_{0.85} \mathrm{Sr}_{0.15} \mathrm{CuO}_{2}$. It is considered here first because the bands of this infinite cuprate stack are not perturbed by hybridization with block layer states and hence in this sense are pure. The experimental lattice parameters of $\mathrm{Ca}_{0.85} \mathrm{Sr}_{0.15} \mathrm{CuO}_{2}$ [19] are taken for the fictitious $\mathrm{CaCuO}_{2}$. The antiferromagnetic unit cell is shown in Fig. 1] The space group is $\mathrm{I} 4 / \mathrm{mmm}$ (139). The distance along $\mathrm{Cu}-\mathrm{O}-\mathrm{Cu}$ is $d_{a}=3.86 \AA$ and the distance in $z$-direction between adjacent $\mathrm{CuO}_{2}$ layers is $d_{c}=3.20 \AA$. (For all compounds considered here $d_{a}$ is the lattice constant of the non-magnetic cuprate plane, while $d_{c}$ is the distance in $z$-direction between adjacent cuprate multilayers $\left[\left(\mathrm{CuO}_{2}\right)_{n} \mathrm{Ca}_{n-1}\right]$. For $\mathrm{CaCuO}_{2}$ it is the $\mathrm{CuO}_{2}$ plane distance. It also gives the periodicity of the multilayers in $z$-direction, ignoring a centering shift perpendicular to the $z$-direction.)

The AFM lattice constants are $a_{0}=b_{0}=\sqrt{2} d_{a}, c_{0}=2 d_{c}$. The atom positions are $\mathrm{Ca}\left(0, \frac{1}{2}, \frac{1}{4}\right), \mathrm{Cu}(0,0,0), \mathrm{Cu}\left(0,0, \frac{1}{2}\right)$ and $\mathrm{O}\left(\frac{1}{4}, \frac{1}{4}, 0\right)$. Table 1 gives the FPLO basis set. The number of Fourier components was 1024 per atom and the k-mesh subdivision was $(6,6,6)$. Nonrelativistic calculations were performed.

The symmetry points of the band structures presented below refer to the Brillouin zone of the antiferromagnetic cuprate plane (a square of edge length $\left.2 \pi /\left(\sqrt{2} d_{a}\right)\right)$. For better comparison to the literature we relate it to the nonmagnetic Brillouin zone which is a rotated by $45^{\circ}$ square of edge length $2 \pi / d_{a}$. In units of $\pi / d_{a}$ we have $\Gamma,(Z)=(0,0, \zeta), X,(R)=\left(\frac{1}{2}, \frac{1}{2}, \zeta\right)$ and $M,(A)=(1,0, \zeta)$. The first labels refer to $\zeta=0$ and the labels in parentheses refer to $\zeta=1 \hat{=} \pi / d_{c}$. (This is the $Z$ point of a simple tetragonal cell of lattice constant $c=d_{c}$.)

Calculations within LSDA and LSDA $+U$ were performed. As usual for the cuprates, the LSDA gives a metallic ground state. The KS bandstructure is shown in Fig. 3. The spaghetti below the Fermi level (here and in all following figures put equal to zero) consists of hybridized $\mathrm{Cu}-3 d$ and $\mathrm{O}-2 p$ states, with the bonding combinations at the bottom and the antibonding bands formed of the orbitals of Fig. 2 crossing the Fermi level. Non-bonding combinations are in between. The unoccupied bands above the Fermi level start with $\mathrm{Cu}-4 s$ and $\mathrm{Cu}-4 p$ character and then enter a bunch of Ca-3d bands above $5 \mathrm{eV}$.

The same bands weighted (by linewidth) with the square of the coefficient of selected basis orbitals in the KS state are shown in Fig. 4. From the upper two panels one can read off a $(\mathrm{Cu}-\mathrm{O})_{\sigma}$ covalency split of more than $5 \mathrm{eV}$ while the third panel shows a $(\mathrm{O}-\mathrm{O})$ covalency split of about $3 \mathrm{eV}$. One further observes that the $\mathrm{O}_{\sigma}$ orbitals and the $\mathrm{O}_{z}$ orbitals hybridize also with the $\mathrm{Cu} 4 s$ and $4 p$ orbitals while the in-plane $\mathrm{O}_{\pi}$ orbitals hybridize additionally with the Ca $3 d$ orbitals. Figs. 3 and 4 are presented here for comparison with the LSDA $+U$ results shown below.

Experimentally, $\left(\mathrm{Ca}_{0.85} \mathrm{Sr}_{0.15}\right) \mathrm{CuO}_{2}$ is an AFM insulator with a bandgap of more than $1 \mathrm{eV}$ and a Néel temperature $T_{N} \approx 540 \mathrm{~K}$. The $U$-functional cures 
this deficiency and one finds an AFM solution with a spin polarization energy $\Delta E=27.9 \mathrm{mH}$ a per formula unit below the Pauli-paramagnetic (PM) state. The site projected copper $3 d$ moment is $0.71 \mu_{B}$ and the total copper spin moment is $0.69 \mu_{B}$ (reduced by negative $3 s 3 p$ moments).

The already discussed two relevant molecular orbitals (MO) of Fig. 2 are the candidates for the highest occupied molecular orbital (HOMO) in the correlated electronic structure of cuprates, that is, those MOs which are relevant for the valence band edge. The LSDA $+U \mathrm{KS}$ bands of $\mathrm{CaCuO}_{2}$ with the AMF and AL functionals are shown in Fig. [5] The main difference between both functionals is found in the unoccupied bands. As to be expected for a more than half-filled shell, the upper Hubbard band of $\left(\mathrm{Cu}-3 d_{x^{2}-y^{2}}\right.$ and $\mathrm{O}-2 p_{\sigma}$ character, see below) lies higher in the AMF case compared to AL. In AL this band is the lowest unoccupied band. In the occupied part near the Fermi level the differences are small, while far below larger differences are found due to the different position of the lower Hubbard band and thus to different hybridization. Since this paper focuses on the occupied bands near the Fermi level, the differences are not very relevant, and all further results are presented for the AMF functional.

On Fig. [6 the orbital weights to the AMF bands in analogy to Fig. 4] is shown. First, it is clearly seen that the upper and lower Hubbard band is formed by $\mathrm{Cu}-3 d_{x^{2}-y^{2}}$ and $\mathrm{O}-2 p_{\sigma}$ orbitals. At the valence band edge (points $\mathrm{X}$ and $\mathrm{R}$ shown, but without noticeable dispersion on the whole line $\mathrm{X}-\mathrm{R}$ in $z$-direction of the $\boldsymbol{k}$-space) $\mathrm{O}-2 p_{\sigma}$ and $\mathrm{O}-2 p_{\pi}$ contribute equally strongly while the $\mathrm{Cu}-3 d_{x^{2}-y^{2}}$ orbital contribution is largely suppressed compared to the LSDA result. This suppression of the $\mathrm{Cu}$ contribution to valence holes in cuprates is confirmed by experiment 24]. The O- $2 p_{\pi}$ contribution on the other hand is strongly enhanced compared to LSDA, a new result which is missed in most model Hamiltonian treatments in the literature where the $\mathrm{O}-2 p_{\pi}$ degree of freedom is excluded from the Hamiltonian in most cases. Other orbitals do not contribute. Hence, on the line X-R the highest occupied band is mainly a hybrid of O- $2 p_{\sigma}$ and O- $2 p_{\pi}$. These band states are assumed to form the Zhang-Rice singlet with the nominal $3 d$-hole on the $\mathrm{Cu}$ site (upper Hubbard state) 21]. The bandwidth of the highest valence band is about $1 \mathrm{eV}$ due to hybridization with a flat band $1 \mathrm{eV}$ below the Fermi level. On the line X-M (R-A) the $\mathrm{O}-2 p_{\sigma}$ contribution fades away towards $\mathrm{M}(\mathrm{A})$ due to this hybridization. In LSDA the $\mathrm{O}-2 p_{\pi}$ orbitals do practically not contribute to this band which led to the neglect of that orbital in model Hamiltonians. This failure of the LSDA is mainly due to the fact that in LSDA the $\mathrm{O}-2 p_{\pi}$ bands are deeper in energy compared to the $\mathrm{Cu}-3 d_{x^{2}-y^{2}}-\mathrm{O}-2 p_{\sigma}$ bands.

The absolutely highest occupied band in the LSDA $+U$ result is a pure O- $2 p_{\pi}$ band (at $\Gamma$ ) which therefore is in energetic competition with the Zhang-Rice state when adding additional holes. The $\mathrm{O}-2 p_{\pi}$ bands show a considerable dispersion in $z$-direction which comes from a weak hybridization with the unoccupied Ca$3 d_{x^{2}-y^{2}}$ orbitals. The $\mathrm{Cu} 4 s$ orbitals do not contribute at the valence band edge. They are mixed into the unoccupied states and are also slightly mixed into O- $2 p_{\sigma}$ 
states about $1.5 \mathrm{eV}$ below the Fermi level on the line M-A.

\section{$3.2 \quad \mathrm{Sr}_{2} \mathrm{CuO}_{2} \mathrm{Cl}_{2}$}

The single layer compound $\mathrm{Sr}_{2} \mathrm{CuO}_{2} \mathrm{Cl}_{2}$ is probably the most two-dimensional of all cuprates. The block layer consists of $2 \mathrm{SrCl}$ layers which separate single $\mathrm{CuO}_{2}$ planes. Between these $\mathrm{SrCl}$ layers crystals are easily cleaved, whence most photoemission data on undoped planar cuprates are recorded from this material. Two adjacent cuprate planes are shifted horizontally by a shift vector $\left(\frac{1}{2}, \frac{1}{2}, 0\right)$ relative to each other, which produces a body centered non-magnetic unit cell. The large distance of cuprate planes from each other prevents valence state coupling in $z$-direction. The AFM cell has base centered orthorhombic symmetry and is shown in Fig. 7

The standard cell choice for the orthorhombic cell, which has also to be used in the FPLO code results in c-base centering. (The stacking direction is the $b$-direction and the cuprate plane is the $c, a$ plane.) The space group is Cmmm (65). The cell parameters are $d_{a}=3.973 \AA$ and $2 d_{c}=15.618 \AA[25$. The lattice constants of the AFM cell are $a_{0}=c_{0}=\sqrt{2} d_{a}, b_{0}=2 d_{c}$. The atom positions are $\mathrm{Cu}\left(0, \frac{1}{2}, \frac{1}{2}\right), \mathrm{Cu}(0,0,0), \mathrm{O}\left(-\frac{1}{4}, 0,-\frac{1}{4}\right), \mathrm{Cl}\left(0,-0.317, \frac{1}{2}\right), \mathrm{Cl}$ $(0,0.183,0), \operatorname{Sr}\left(0,-0.107, \frac{1}{2}\right)$ and $\operatorname{Sr}(0,0.393,0)$. Table 2 gives the basis set. The number of Fourier components was 1000 per atom and the k-mesh subdivision was $(12,12,12)$. Scalar relativistic calculations are performed. For the sake of comparison the stacking direction is further on denoted $z$ and the cuprate plane as the $x, y$-plane. The symmetry points are chosen corresponding to the scheme described in the previous subsection.

The site projected copper $3 d$ moment of the LSDA $+U$ result is $0.758 \mu_{B}$ and the total copper spin moment is $0.748 \mu_{B}$. In Fig. 8 the orbital weights for the relevant bands are shown. The situation is rather similar to that of $\mathrm{CaCuO}_{2}$, so only the differences are pointed out.

The "Zhang-Rice" band is slightly higher and touches the Fermi level at X and $\mathrm{R}$. The intersecting band is correspondingly lower at the line M-A, as a consequence the width of the upper valence band is about $1.5 \mathrm{eV}$ and the fading of the $\mathrm{O}-2 p_{\sigma}$ character on the lines X-M and R-A is less pronounced, a reduction of about 50 p.c. remains. The $z$-dispersion of the $\mathrm{O}-2 p_{\pi}$ band on the line $\Gamma$-Z has gone as there are no Ca- $3 d$ states present for hybridization. There is a marked $2 \mathrm{D}$ character of the compound.

The quasiparticle low energy dispersion measured by ARPES (single hole excitation) is shown in Fig. 9. A detailed discussion is found in 26] and 27. The left part of the experimental spectra (from $(0,0)$ to $\left(\frac{\pi}{2}, \frac{\pi}{2}\right)$ ) compares nicely with the $\mathrm{O}-2 p_{\sigma}$ dominated LSDA $+U$ band on the line $\Gamma-X$ (second panel of Fig. (8) and the right part (from $\left(\frac{\pi}{2}, \frac{\pi}{2}\right)$ to $(0, \pi)$ ) with the same LSDA $+U$ band on the line $X-M$. Even the reported fading ARPES intensity when going from $X$ towards $M$ (see also [28]) agrees with the fading O- $2 p_{\sigma}$ projection of that band. 
Nevertheless, the experimental band width is smaller by a factor of about two to three and the situation on the line $\Gamma-M$ is less clear although the comparison of only the O- $2 p_{\sigma}$ projected bands (second panel of Fig. 8] cf. the discussion of models above) to model results contained in Fig. 9 is not so bad. After all, LSDA $+U$ accounts for electron correlations still rather grossly.

\section{$3.3 \quad \mathrm{Bi}_{2} \mathrm{Sr}_{2} \mathrm{CaCu}_{2} \mathrm{O}_{8}$}

The bilayer compound $\mathrm{Bi}_{2} \mathrm{Sr}_{2} \mathrm{CaCu}_{2} \mathrm{O}_{8}$ has a double layer of cuprate planes separated by block layers. The block layer consists of two $\mathrm{BiSrO}_{2}$ layers between which crystals cleave equally easily as in the previous case and also favor the material for photoemission. Adjacent double layers are again shifted horizontally by a shift vector $\left(\frac{1}{2}, \frac{1}{2}, 0\right)$ relative to each other, which produces a body centered tetragonal non-magnetic cell. The hypothetic AFM cell again is assumed base centered orthorhombic.

The cell parameters are again given in the c-base centered setting, resulting in the $b$-axis being the stacking direction. The space group is Cmmm (65). The cell parameters are $d_{a}=3.817 \AA$ and $2 d_{c}=30.6 \AA$. The lattice constants of the AFM cell are $a_{0}=c_{0}=\sqrt{2} d_{a}, b_{0}=2 d_{c}$. The atom positions are Ca $\left(0, \frac{1}{4} \frac{1}{4}\right)$, Sr $\left(0,0.3597, \frac{1}{4}\right), \operatorname{Sr}\left(0,0.1403, \frac{1}{4}\right), \mathrm{Bi}\left(0,-0.4478, \frac{1}{4}\right), \mathrm{Bi}\left(0,-0.0522, \frac{1}{4}\right)$, $\mathrm{Cu}\left(0,-0.3040, \frac{1}{4}\right), \mathrm{Cu}\left(0,-0.1960, \frac{1}{4}\right), \mathrm{O}_{1}\left(-\frac{1}{4}, 0.1960,0\right), \mathrm{O}_{2}\left(0,-0.1250, \frac{1}{4}\right), \mathrm{O}_{2}$ $\left(0,-0.3750, \frac{1}{4}\right), \mathrm{O}_{3}\left(0,0.0450, \frac{1}{4}\right)$ and $\mathrm{O}_{3}\left(0,0.4550, \frac{1}{4}\right)$. The two $\mathrm{Sr}, \mathrm{Bi}, \mathrm{O}_{2}, \mathrm{O}_{3}$ and $\mathrm{Cu}$ atoms are equivalent in the non-magnetic cell. The $\mathrm{O}_{1}$ atom is that of the cuprate plane. The two $\mathrm{Cu}$ spins are antiferromagnetically ordered in the AFM cell. Table 3 gives the basis set. The number of Fourier components was 500 per atom and the k-mesh subdivision was $(8,8,8)$. Again, scalar relativistic calculations were performed and the symmetry points in $\boldsymbol{k}$-space are chosen corresponding to the scheme described in subsection 3.2.

For this material both the LSDA and LSDA $+U$ yield a metallic ground state with $\mathrm{Cu}-\mathrm{O}$ bands and $\mathrm{Bi}$ bands crossing the Fermi level. While the LSDA results in a non-magnetic solution, the LSDA $+U$ calculation yields a stable AFM state. The site projected copper $3 d$ moment is $0.696 \mu_{B}$ and the total copper spin moment is $0.684 \mu_{B}$.

Fig. 10 shows both the LSDA and LSDA $+U$ bands of $\mathrm{Bi}_{2} \mathrm{Sr}_{2} \mathrm{CaCu}_{2} \mathrm{O}_{8}$ close to the Fermi level. For a better orientation in the band character the $\mathrm{Cu}-3 d_{x^{2}-y^{2}}$ projected bands are also shown. Note that the LSDA $+U$ results on the right panels have twice as many bands as the LSDA results on the left panels due to the AFM order of the former ground state. The bands crossing the Fermi level and not seen in the lower panels are $\mathrm{Bi}$ bands (more precisely $\mathrm{BiO}$ bands hybridized with orbitals of the block layer oxygen).

The splitting of the Cu-3 $d_{x^{2}-y^{2}}$ projected LSDA bands crossing the Fermi level on the line X-M (R-A) is the much discussed bilayer splitting between bonding and antibonding combinations of the $\mathrm{CuO}$ states in both $\mathrm{CuO}_{2}$ layers of the 
bilayer [27. The coupling of those states is mainly due to a small hybridization with $\mathrm{Cu}-4 s$ states, and this part of the coupling has $k_{x}^{2}-k_{y}^{2}$ symmetry. Hence, the splitting is maximum $(\sim 0.25 \mathrm{eV})$ at point $\mathrm{M}(\mathrm{A})$ and nearly zero at point $\mathrm{X}(\mathrm{R})$ although a very small splitting remains there due to a small direct coupling. It is readily seen by a simple symmetry argument that in the AFM state this splitting must be zero on the whole line X-M (R-A), if spin-orbit coupling is neglected. Accordingly all bands on the right panels of Fig. 10 are twofold degenerate on these lines and do not show a bilayer splitting there. This only develops away from these lines, for instance in the $\mathrm{X}-\Gamma$ and $\mathrm{M}-\Gamma$ directions. However, the $\mathrm{Cu}-$ $3 d_{x^{2}-y^{2}}$ projected LSDA $+U$ bands on the right lower panel show around $\mathrm{M}$ and A another splitting of approximately the same magnitude which is due to the crossing of another oxygen band. This point has never been considered in the literature to date.

Note also that the non-magnetic structure of the left panels has a larger Brillouin zone so that the rising band from $\Gamma$ to $\mathrm{X}$ continues to rise from $\mathrm{X}=\left(\frac{1}{2}, \frac{1}{2}, 0\right)$ to the Brillouin zone corner $(1,1,0)$, whereas the AFM structure of the right panels has a charge transfer gap (dominated by $U$ and hence much larger than the exchange splitting) at X, which now lies on the Brillouin zone boundary, and the conduction band has its maximum at X. On the other hand, the band pair close to the Fermi level on the line $\Gamma-Z$ on the upper right panel is on the line $(1,1, \zeta)$ in the non-magnetic state and not shown on the upper left panel. To illustrate the charge transfer gap, the LSDA $+U$ band structure projected on $\mathrm{Cu}-3 d_{x^{2}-y^{2}}$ is shown once more in Fig. 11 for a larger energy window.

Experimentally, the oxygen in the block layers of $\mathrm{Bi}_{2} \mathrm{Sr}_{2} \mathrm{CaCuO}_{8}$ is volatile and its stoichiometry is governed by thermodynamics. Moreover, the geometry of the block layer is distorted in a disordered way compared to the ideal structure used in the calculation. In the recent, highest resolution photoemission spectra [27, Fermi surface pockets around point $M$ which should be present due to the $\mathrm{Bi}$ bands in both the LSDA and LSDA $+U$ results are not seen. Hence, one could assume that they are pushed away from the Fermi level (together with the oxygen bands on $\Gamma$-Z) by a distortion potential. Correspondingly, the Fermi level would be lowered to ensure the electron count. This would be a big problem for the LSDA band structure where not only the antibonding bilayer split band would have an electron Fermi surface closed around $\Gamma$ but also the bonding one, both in contradiction to what is seen in photoemission. The LSDA $+U$ band structure (lower right panel of Fig. (10) on the contrary would be in rather good agreement with photoemission if one wipes out the down folded bands of the AFM order which is not found in experiment. (The material cannot be reduced down to undoped $\mathrm{CuO}_{2}^{2-}$ planes.)

There would be another stark difference to what is discussed in the literature with respect to the bilayer splitting: If the band splitting around $\mathrm{M}$ and observed in photoemission would be a bilayer splitting, one should expect it to be strongly reduced when reducing the doping level to an underdoped superconductor which 
is regarded to develop strong AFM correlations (also seen in neutron scattering). It should be reduced to zero where AFM order sets in. The splitting of the $\mathrm{LSDA}+U$ bands of different origin on the contrary is to be expected largely independent of antiferromagnetic order and hence on doping.

\subsection{Implications on Magnetic Interactions}

Magnetic couplings may grossly be obtained from total energy differences of $\mathrm{LSDA}+U$ results for ferromagnetic and antiferromagnetic order; in more detail they may be obtained from calculated energies of spin spiral states [29].

For a more detailed understanding of their physics, a tight-binding model of the kind of Emery's model should be extracted from the LSDA $+U$ results, which then may be down-mapped to a kind of a $t-J$ model. For a hypothetic ferromagnetic order (assumed for the sake of simplicity) such a tight-binding model was derived in Ref. [23].

One main conclusion from the present orbital analysis is that in a large part of the Brillouin zone there is a strong hybridization of O- $2 p_{\sigma}$ with $\mathrm{O}-2 p_{\pi}$ orbitals in bands hybridized with the $\mathrm{Cu}-3 d_{x^{2}-y^{2}}$ orbital. Furthermore, the $\mathrm{O}-2 p_{\pi}$ bands are in energetic competition with the bands forming the Zhang-Rice state, if additional holes are doped. Hence, the $\mathrm{O}-2 p_{\pi}$ orbitals must be included in the Emery model in order to correctly describe the $t$-terms which determine the magnetic coupling.

In $\mathrm{CaCuO}_{2}$ there is a sizable dispersion of the in-plane $\mathrm{O}-2 p_{\pi}$ bands in $z$ direction mediated by some hybridization with Ca- $3 d_{x^{2}-y^{2}}$ orbitals. This is in accordance with the experimental finding that $\mathrm{CaCuO}_{2}$ has the highest Néel temperature, $T_{N}=540 \mathrm{~K}$, of all layered cuprates indicating 3D magnetism [19. 30. For a survey of the magnetic properties of cuprates see Ref. 31.

By contrast, in $\mathrm{Sr}_{2} \mathrm{CuO}_{2} \mathrm{Cl}_{2}$ there is no dispersion of the corresponding bands in $z$-direction due to the $\mathrm{Sr}_{2} \mathrm{Cl}_{2}$ buffer layers. There is only dipole-dipole coupling of the planes, compatible with the experimental findings, $T_{N}=256 \mathrm{~K}[32$.

\section{Conclusions}

The LSDA $+U$ approach is shown to fit perfectly in the frame of DFT by Hohenberg and Kohn for the electronic ground state, provided the theory can be closed by linking the $U$-value to the variational quantities, the KS orbitals and orbital occupation numbers (which explicit link is yet to be rendered). On the other hand, the LSDA $+U$ potential is also widely understood as an approximation to the electron self-energy (first step towards an LSDA+dynamical mean-field theory). There is hope that in this way like in the situation of weakly correlated systems it provides again a tool to obtain a rather accurate ground state and a reasonable approximation to the quasi-particle spectrum (band structure) in a single run from only one set of equations. To pursue this goal, in the first part of 
the present paper an attempt was made to present the structure of the LSDA $+U$ theory as clearly as possible.

Application to several typical planar cuprate structures and comparison to experimental data of the isolating electronic state and the magnetic state as well as of quasi-particle spectra probed by photoemission seems to support this expectation. On the other hand it revealed a number of new aspects in the physics of the electronic structure of cuprates. Notably the sufficient completeness of most model Hamiltonians in use must be questioned, at least in connection with the dimensionality and details of magnetic couplings, and the so-called bilayer splitting of the band structure of $\mathrm{Bi}_{2} \mathrm{Sr}_{2} \mathrm{CaCu}_{2} \mathrm{O}_{8}$ should be reanalyzed.

\section{Acknowledgments}

We thank H. Rosner for helpful discussions. Financial support by the German Israel Foundation under Contract No. I-614-13.14/99 and by the Deutsche Forschungsgemeinschaft, SFB 463, is gratefully acknowledged.

\section{References}

[1] P. Hohenberg and W. Kohn, Phys. Rev. 136, B864-71 (1964); W. Kohn and L. J. Sham, Phys. Rev. 140, A1133-A1138 (1965).

[2] E. H. Lieb, Int. J. Quant. Chem. XXIV, 243-77 (1983).

[3] H. Eschrig "The Fundamentals of Density Functional Theory", B. G. Teubner, Stuttgart, 1996; a revised and extended issue is in preparation by Stiftung Benedictus Gotthelf Teubner, Leipzig.

[4] J. F. Herbst, R. E. Watson and J. W. Wilkins, Phys. Rev. B17, 3089-98 (1978).

[5] P. H. Dederichs et al., Phys. Rev. Lett. 53, 2512-15 (1984).

[6] M. R. Norman and A. J. Freeman, Phys. Rev. B33, 8896-98 (1986).

[7] O. Gunnarsson et al., Phys. Rev. B39, 1708-22 (1989).

[8] W. E. Pickett and C. S. Wang, Phys. Rev. B30, 4719-33 (1984).

[9] M. S. Hybertsen and S. G. Louie, Phys. Rev. Lett. 55, 1418-21 (1985).

[10] V. I. Anisimov, J. Zaanen and O. K. Andersen, Phys. Rev. B44, 943-54 (1991).

[11] V. I. Anisimov et al., Phys. Rev. B48, 16 929-34 (1993).

[12] M. T. Czyżyk and G. A. Sawatzky, Phys. Rev. B49, 14211-28 (1994).

[13] V. I. Anisimov, F. Aryasetiawan and A. I. Lichtenstein, J. Phys.: Condens. Matter 9, 767-808 (1997). 
[14] A. B. Shick, A. I. Liechtenstein and W. E. Pickett, Phys. Rev. B60, 10763-69 (1999).

[15] K. Koepernik and H. Eschrig, Phys. Rev. B59, 1743-57 (1999).

[16] A. I. Liechtenstein, V. I. Anisimov and J. Zaanen, Phys. Rev. B52, R5467-70 (1995).

[17] Access to FPLO exists under http://www.ifw-dresden.de/fplo.

[18] P. Perdew and A. Zunger, Phys. Rev. B23, 5048-79 (1981).

[19] D. Vaknin et al., Phys. Rev. B39, 9122-25 (1989).

[20] V. J. Emery, Phys. Rev. Lett. 58, 2794-97 (1987).

[21] F. C. Zhang and T. M. Rice, Phys. Rev. B37, 3759-61 (1988).

[22] J. J. M. Pothuizen et al., Phys. Rev. Lett. 78, 717-19 (1997).

[23] R. Hayn et al., Phys. Rev. B60, 645-58 (1999).

[24] J. Fink et al., J. Electron Spectrosc. Relat. Phenom. 66, 395-452 (1994).

[25] L. L. Miller et al., Phys. Rev. B41, 1921-25 (1990).

[26] T. Tohyama, S. Maekawa, Supercond. Sci. Technol. 13, R17-R32 (2000).

[27] A. Damascelli, Z-X. Shen and Z. Hussain, Rev. Mod. Phys., (2003), in press.

[28] F. Ronning et al., cond-mat/0209651 (2002).

[29] A. N. Yaresko et al., Phys. Rev. B65, 115111-1-7 (2002).

[30] R. Pozzi et al., Phys. Rev. B56, 759-65 (1997).

[31] D. C. Johnston In K. H. J. Buschow (ed.) "Handbook of Magnetic Materials", Elsevier, Amsterdam, 1997, Vol. 10, Chap. 1. pp. 1-237.

[32] M. Greven et al., Phys. Rev. Lett. 72, 1096-99 (1994). 


\begin{tabular}{lll}
\hline Atom & core & valence \\
\hline \hline $\mathrm{Ca}$ & $1 s 2 s 2 p$ & $3 s(-1) 3 p(-1) 4 s(1.1240) 4 p(1.0927) 3 d(-1)$ \\
$\mathrm{Cu}$ & $1 s 2 s 2 p$ & $3 s(-1) 3 p(-1) 4 s(1.3148) 4 p(1.2648) 3 d(1.3500)$ \\
$\mathrm{O}$ & $1 s$ & $2 s(1.2869) 2 p(1.2822) 3 d(-1)$ \\
\hline
\end{tabular}

Table 1: Basis set for $\mathrm{CaCuO}_{2}$. (Compression parameter for valence orbitals in parentheses.)

\begin{tabular}{lll}
\hline Atom & core & valence \\
\hline \hline $\mathrm{Cu}$ & $1 s 2 s 2 p$ & $3 s(-1) 3 p(-1) 4 s(1.2300) 4 p(1.1199) 3 d(1.3148)$ \\
$\mathrm{O}$ & $1 s$ & $2 s(1.2580) 2 p(1.2377) 3 d(1.1162)$ \\
$\mathrm{Cl}$ & $1 s 2 s 2 p$ & $3 s(1.1518) 3 p(1.1338) 3 d(1.0852)$ \\
$\mathrm{Sr}$ & $1 s 2 s 2 p 3 s 3 p 3 d$ & $4 s(-1) 4 p(-1) 5 s(1.1360) 5 p(1.0816) 4 d(1.1503)$ \\
\hline
\end{tabular}

Table 2: Basis set for $\mathrm{Sr}_{2} \mathrm{CuO}_{2} \mathrm{Cl}_{2}$. (Compression parameter for valence orbitals in parentheses.)

\begin{tabular}{lll}
\hline Atom & core & valence \\
\hline \hline $\mathrm{Ca}$ & $1 s 2 s 2 p$ & $3 s(-1) 3 p(-1) 4 s(1.1298) 4 p(1.1210) 3 d(1.2286)$ \\
$\mathrm{Sr}$ & $1 s 2 s 2 p 3 s 3 p 3 d$ & $4 s(-1) 4 p(-1) 5 s(1.1239) 5 p(1.0717) 4 d(1.1435)$ \\
$\mathrm{Bi}$ & $1 s 2 s 2 p 3 s 3 p 3 d 4 s 4 p 4 d 4 f$ & $5 s(-1) 5 p(-1) 6 s(1.3570) 6 p(1.2922) 5 d(1.4752)$ \\
$\mathrm{Cu}$ & $1 s 2 s 2 p$ & $3 s(-1) 3 p(-1) 4 s(1.2229) 4 p(1.1549) 3 d(1.3060)$ \\
$\mathrm{O}_{1}$ & $1 s$ & $2 s(1.2464) 2 p(1.2177) 3 d(-1)$ \\
$\mathrm{O}_{2}$ & $1 s$ & $2 s(1.1766) 2 p(1.1895) 3 d(-1)$ \\
$\mathrm{O}_{3}$ & $1 s$ & $2 s(1.0683) 2 p(1.0951) 3 d(-1)$ \\
\hline
\end{tabular}

Table 3: Basis set for $\mathrm{Bi}_{2} \mathrm{Sr}_{2} \mathrm{CaCu}_{2} \mathrm{O}_{8}$. (Compression parameter for valence orbitals in parentheses.) 

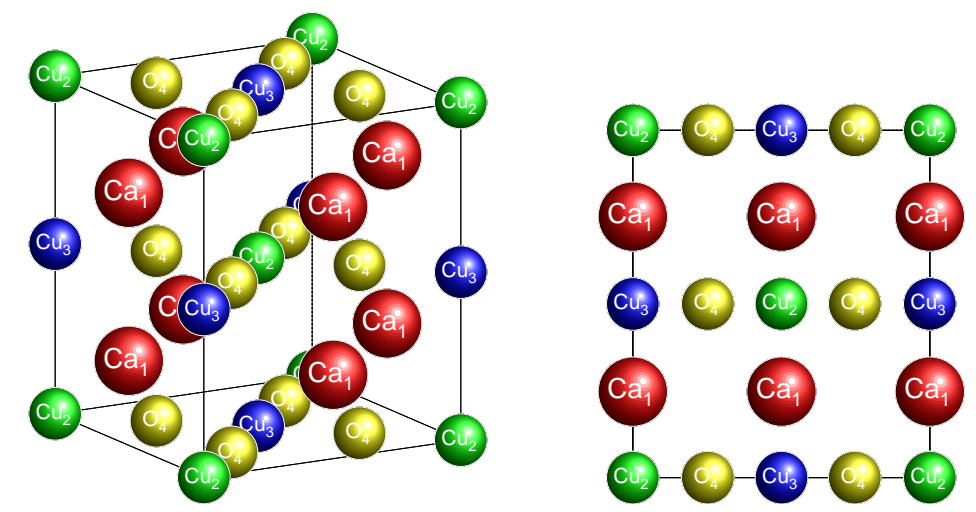

Figure 1: Unit cell of antiferromagnetic $\mathrm{CaCuO}_{2} ; \mathrm{Cu}_{2}$ spin up, $\mathrm{Cu}_{3}$ spin down.
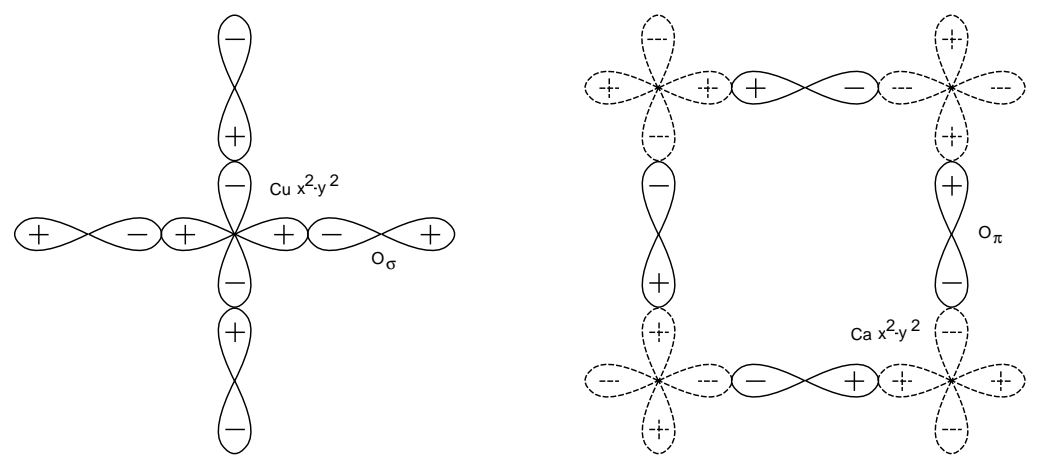

Figure 2: Left: Antibonding $(\boldsymbol{k}=(\pi, \pi, 0)) d p \sigma$-orbital commonly assumed as the HOMO that forms the Zhang-Rice singlet together with the nominal $\mathrm{Cu}-d$ hole [20, 21. Right: O-O antibonding $(\boldsymbol{k}=0)$ in-plane $p \pi$-orbital, lifted up by crystal field and weakly hybridized with $\mathrm{Ca}-d$ orbitals in adjacent layers: the true HOMO of the LSDA $+U$ model 22, 23]. 


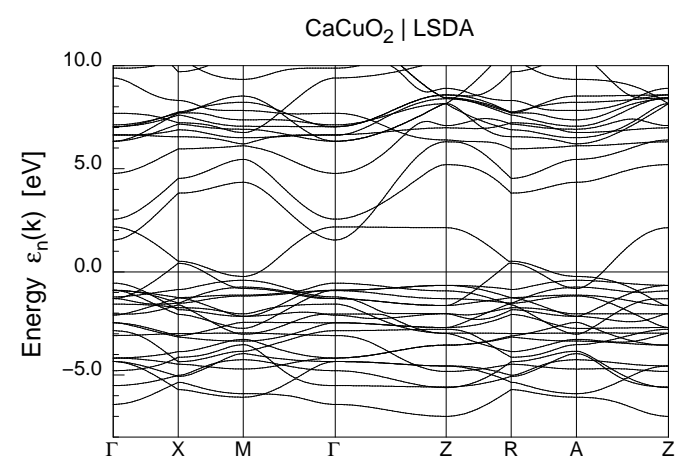

Figure 3: LSDA band structure of $\mathrm{CaCuO}_{2}$. 

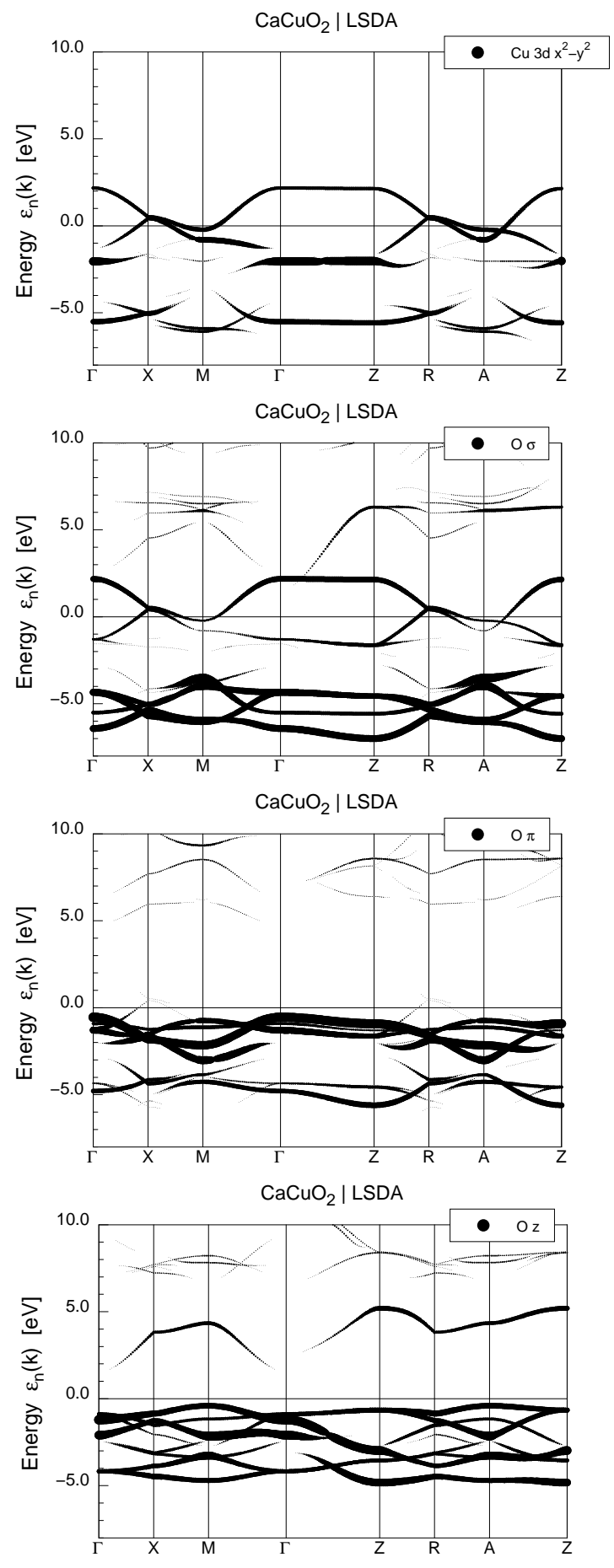

Figure 4: LSDA result for $\mathrm{CaCuO}_{2}$. From top: orbital weight of the $\mathrm{Cu}-3 d_{x^{2}-y^{2}}$ orbital, the $\mathrm{O}-2 p_{\sigma}$ orbitals, the $\mathrm{O}-2 p_{\pi}$ and the $\mathrm{O}-2 p_{z}$ orbitals. 

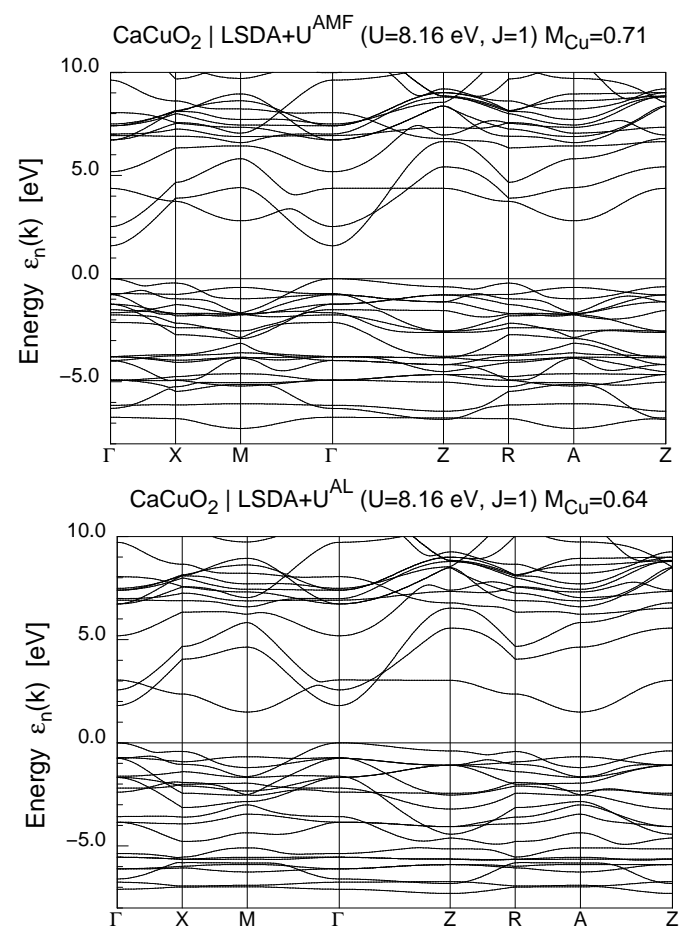

Figure 5: LSDA $+U$ band structure of $\mathrm{CaCuO}_{2}$. Top: AMF, bottom: AL. 

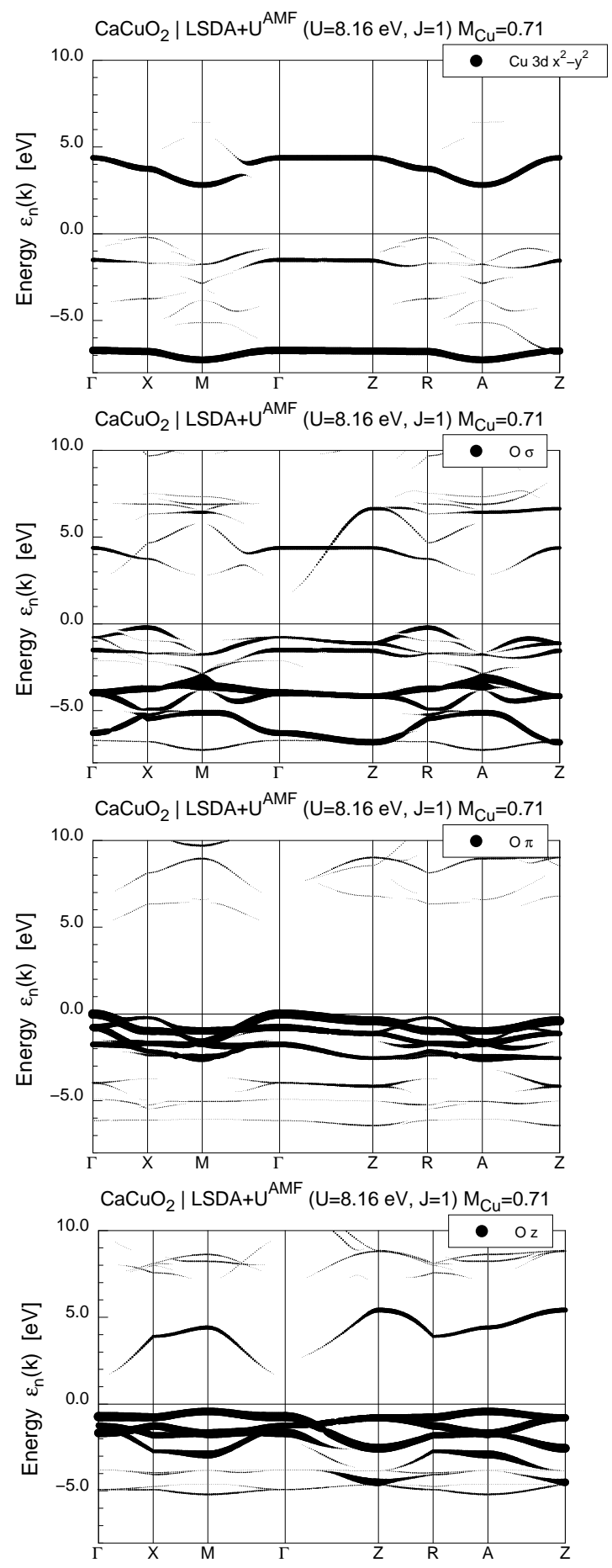

Figure 6: From top to bottom: orbital weight of the $\mathrm{Cu}-3 d_{x^{2}-y^{2}}$ orbital, the $\mathrm{O}-2 p_{\sigma}$ orbitals, the $\mathrm{O}-2 p_{\pi}$ and the $\mathrm{O}-2 p_{z}$ orbitals. 


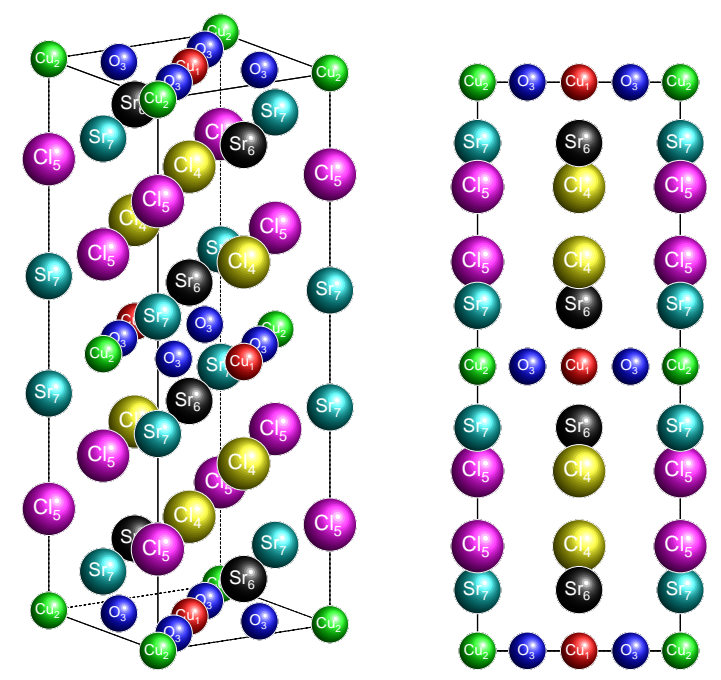

Figure 7: Unit cell of antiferromagnetic $\mathrm{Sr}_{2} \mathrm{CuO}_{2} \mathrm{Cl}_{2} ; \mathrm{Cu}_{1}$ spin up, $\mathrm{Cu}_{2}$ spin down. 

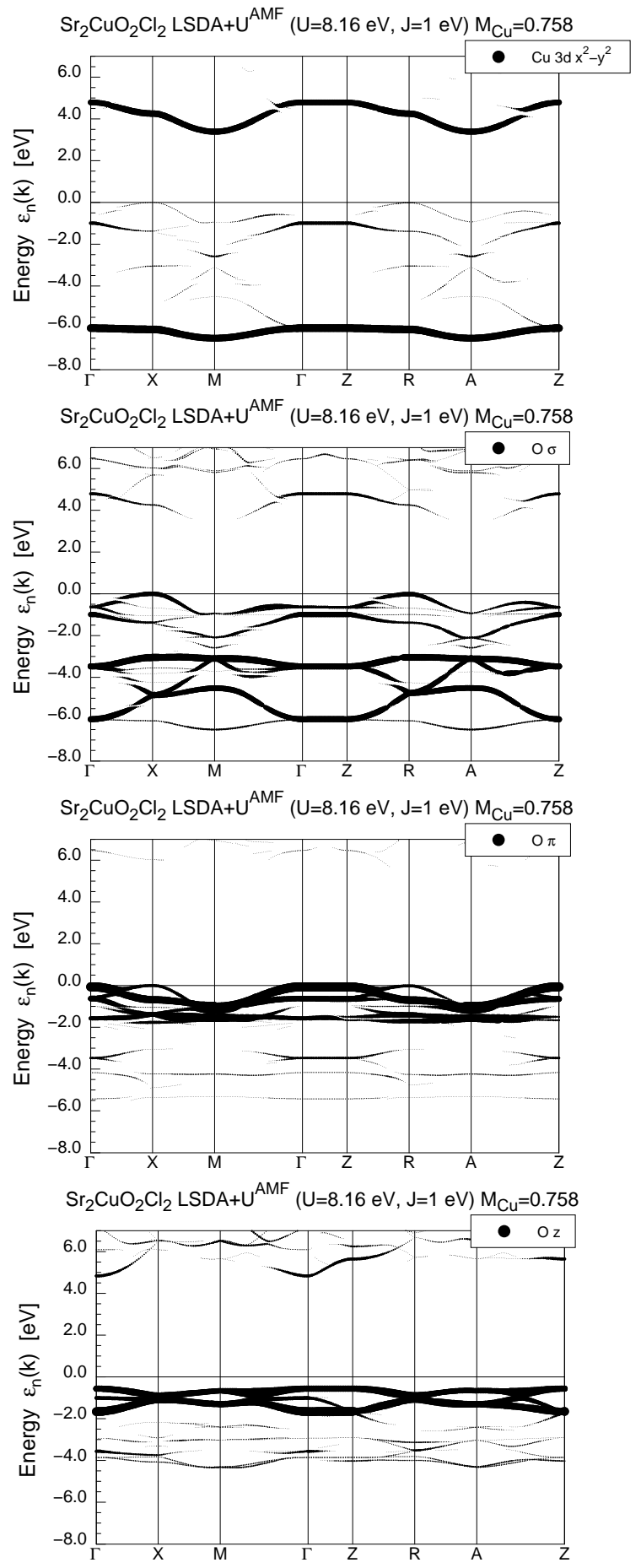

Figure 8: Same as Fig. [6 for $\mathrm{Sr}_{2} \mathrm{CuO}_{2} \mathrm{Cl}_{2}$. 


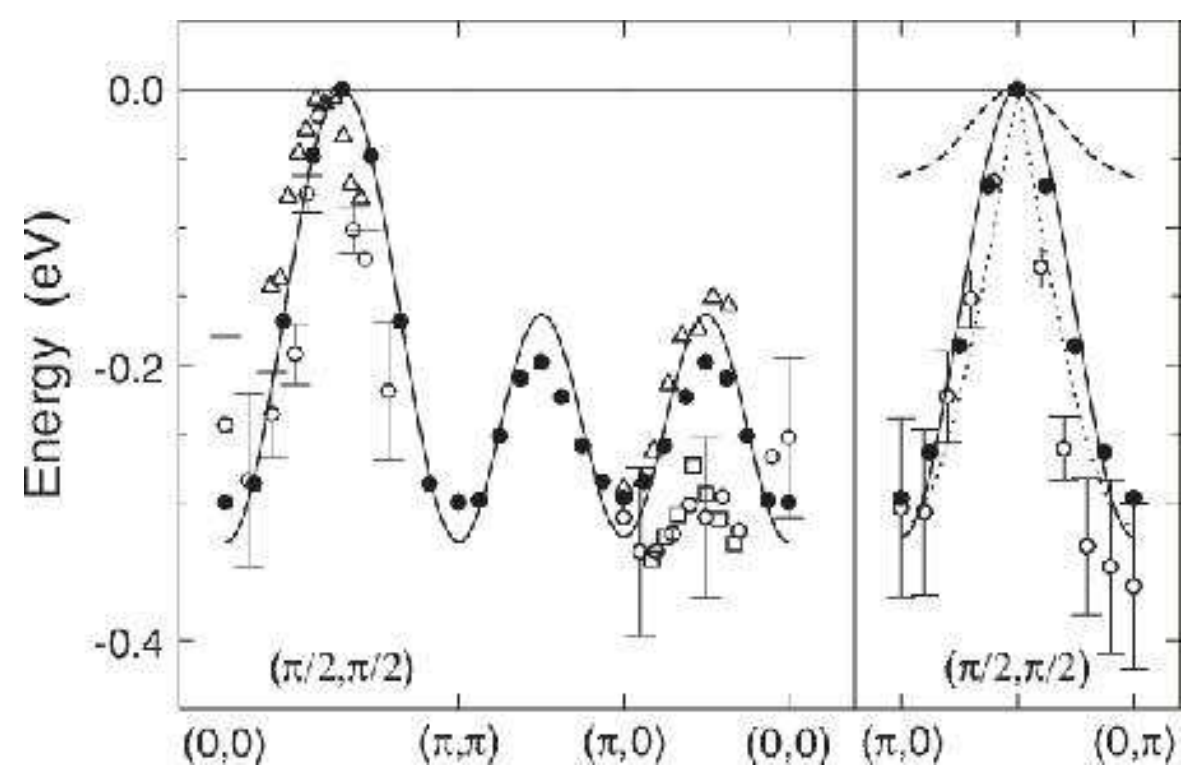

Figure 9: Energy dispersion of quasiparticles for $\mathrm{Sr}_{2} \mathrm{CuO}_{2} \mathrm{Cl}_{2}$ [26]. The energy zero is put at the top of the band, about $0.7 \mathrm{eV}$ below Fermi level. Open symbols: experimental data; solid circles: self-consistent Born approximation for a $t-t^{\prime}-t^{\prime \prime}-J$ model; solid line: tight-binding fit; dashed: $t-J$ model; dotted: spinon model dispersion. 

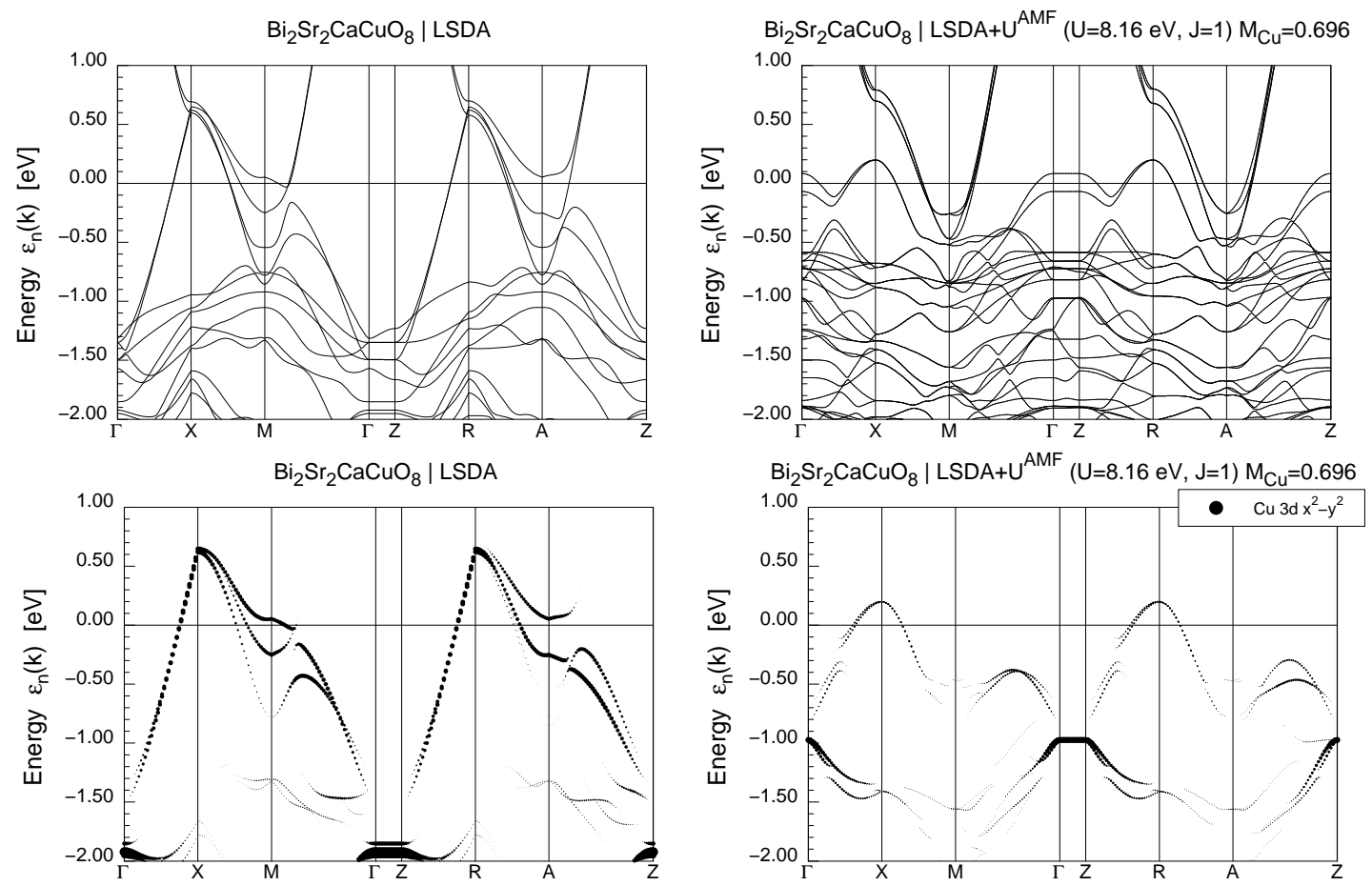

Figure 10: $\mathrm{Bi}_{2} \mathrm{Sr}_{2} \mathrm{CaCu}_{2} \mathrm{O}_{8}$ : left: LSDA band structure and $\mathrm{Cu}-3 d_{x^{2}-y^{2}}$ orbital weights, right: $\mathrm{LSDA}+U^{\mathrm{AMF}}$ bandstructure and weights.

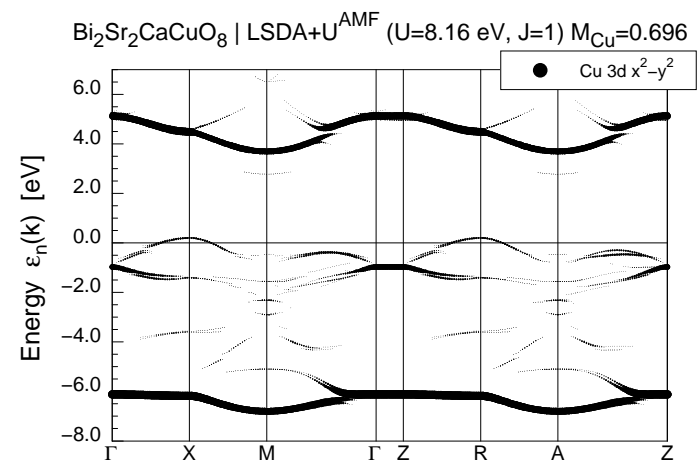

Figure 11: $\mathrm{Bi}_{2} \mathrm{Sr}_{2} \mathrm{CaCu}_{2} \mathrm{O}_{8}$ : LSDA $+U^{\mathrm{AMF}} \mathrm{Cu}-3 d_{x^{2}-y^{2}}$ orbital weights. 